\title{
Crystal structure of a novel cysteinless plant Kunitz-type protease inhibitor
}

\author{
Daiane Hansen ${ }^{a}$, Sandra Macedo-Ribeiro ${ }^{\text {b,c }}$, Paula Veríssimo ${ }^{\text {b }}$, Sonia Yoo Im ${ }^{\text {a }}$, \\ Misako Uemura Sampaio a, Maria Luiza Vilela Oliva ${ }^{\text {a,* }}$ \\ a Departamento de Bioquímica, Universidade Federal de São Paulo-Escola Paulista de Medicina, Rua Três de Maio, 100, 04044-020 São Paulo, SP, Brazil \\ ${ }^{\mathrm{b}}$ Departamento de Bioquímica e Centro de Neurociências e Biologia Celular, Universidade de Coimbra, 3004-517 Coimbra, Portugal \\ ${ }^{\mathrm{C}}$ Instituto de Biologia Molecular e Celular-IBMC, Rua do Campo Alegre 823, 4150-180 Porto, Portugal
}

Received 12 June 2007

Available online 5 July 2007

\begin{abstract}
Bauhinia bauhinioides Cruzipain Inhibitor ( $\mathrm{BbCI}$ ) is a cysteine protease inhibitor highly homologous to plant Kunitz-type inhibitors. However, in contrast to classical Kunitz family inhibitors it lacks cysteine residues and therefore disulfide bridges. BbCI is also distinct in the ability to inactivate enzymes belonging to two different classes, cysteine and serine proteases. Besides inhibiting the cysteine protease cruzipain, BbCI also inhibits cathepsin L and the serine proteases HNE (human neutrophil elastase) and PPE (porcine pancreatic elastase). Monoclinic crystals of the recombinant inhibitor that diffract to $1.7 \AA$ resolution were obtained using hanging drop method by vapor diffusion at $18^{\circ} \mathrm{C}$. The refined structure shows the conservative $\beta$-trefoil fold features of the Kunitz inhibitors. In BbCI, one of the two characteristic S-S bonds is replaced by the water-mediated interaction between Tyr125 and Gly132. In this work we explore the structural differences between Kunitz-type inhibitors and analyze the essential interactions that maintain the protein structural stability preserving its biological function.
\end{abstract}

(C) 2007 Elsevier Inc. All rights reserved.

Keywords: Cathepsin; Crystallography; Cruzipain; Elastase; Kallikrein; Kunitz protease inhibitors; X-ray diffraction

Protease inhibitors have been isolated from diverse sources and they have been associated with diverse events as plant evolution $[1,2]$ and protection against microorganism invasion and insect attack $[3,4]$. Among the many classes of plant inhibitors, the Kunitz family is the best characterized probably due their abundance in seeds $[1,2]$.

Extracts from some Bauhinia species are used in traditional medicine for the diabetes treatment $[5,6]$. We previously isolated an inhibitor from seeds of Bauhinia bauhinioides, Cesalpinoideae, a plant known in Brazil by the trivial name of "cow paw" due the shape of its leaves [7]. This inhibitor was named BbCI, Bauhinia bauhinioides Cruzipain Inhibitor [8], since it inhibits cruzipain, the major cysteine protease isolated of Trypanosoma cruzi

\footnotetext{
* Corresponding author. Fax: +551155723006.

E-mail address: olivaml.bioq@epm.br (M.L.V Oliva).
}

and cruzain $\left(\mathrm{Ki}_{\text {app }} 0.3 \mathrm{nM}\right)$, its recombinant form [9]. Trypanosoma cruzi is the causative agent of Chagas' disease, the parasitic infection that remains as one of the leading causes of heart disease in Latin America [10].

Most curiously, this BbCI shows inhibitory properties against enzymes of two different classes, cysteine and serine proteases. We have verified that $\mathrm{BbCI}$ inhibits porcine pancreatic elastase $\left(\mathrm{Ki}_{\text {app }} 40 \mathrm{nM}\right)$, and human neutrophil elastase $\left(\mathrm{Ki}_{\text {app }} 5.3 \mathrm{nM}\right)$, a key enzyme involved in inflammatory processes $[8,11]$. In vitro, BbCI also inhibits cathepsin $\mathrm{L}\left(\mathrm{Ki}_{\text {app }} 2.2 \mathrm{nM}\right)$ [8], and in contrast, it does not affect the activity of cathepsin B, cathepsin $\mathrm{V}$ and cathepsin $\mathrm{X}$, very similar papain-like proteases [8].

We previously reported a Bauhinia bauhinioides Kallikrein Inhibitor, BbKI, an $18 \mathrm{kDa}$ protein isolated from the same plant seeds, that although displaying a high $(84 \%)$ primary structure identity, differs from BbCI by inhibiting plasma kallikrein $\left(\mathrm{Ki}_{\text {app }} 2.4 \mathrm{nM}\right)$ and plasmin 
$\left(\mathrm{Ki}_{\text {app }} 33 \mathrm{nM}\right)[12,13]$. Those inhibitory properties demonstrated by these atypical Kunitz inhibitors were confirmed by the recombinant proteins [14].

One of the distinct features of the plant Kunitz (STI) family is the presence of two highly conserved disulfide bridges. We have characterized a new subclass of Kunitz inhibitor with only one disulfide bridge $[15,16]$ or no disulfide bridges [17]. The BbCI primary structure presents no cysteine residues, and BbKI bears only one cysteine residue in the $\mathrm{C}$-terminal region. The absence of cysteine residues distinguishes $\mathrm{BbCI}$ from classical Kunitz inhibitors where the disulfide bridges contribute to their stability and biological activity.

Here, we report the three-dimensional structure of the recombinant $\mathrm{BbCI}$ to $1.70 \AA$ resolution and compare it to the structure of the highly homologous BbKI. The threedimensional structures of only a few plant Kunitz-type inhibitors have been determined and they all display a $\beta$-trefoil structure [18-20], composed of a six-stranded $\beta$-barrel closed at one end by three $\beta$-hairpins and exhibiting a characteristic pseudo 3-fold axis of symmetry when viewed down the $\beta$-barrel axis [21]. All representative proteins of the $\beta$-trefoil family display the same 3 -fold repetition as a distinctive structural signature although they are less than $30 \%$ identical at the amino acid sequence level [22].

To the best of our knowledge, this is the first report of a crystallographic structure of an inhibitor of the Kunitz (STI) family without disulfide bridges. Given the importance of the covalent bonds in the stabilization of the reactive site loop $[23,24]$, it was important to elucidate the $\mathrm{rBbCI}$ crystallographic structure in order to understand the structural aspects surrounding its reactive site.

\section{Results and discussion}

\section{rBbCI structure displays a beta-trefoil fold}

rBbCI structure contains 164 residues, numbered from Met (0) to Thr (163). All residues were unambiguously resolved by electron density maps. As in contrast to classical Kunitz-type inhibitors, BbCI has no cysteine residues and therefore no disulfide bridges, in order to further understand the detailed structural features of this cysteinless inhibitor we have solved the crystal structure of $\mathrm{rBbCI}$ at $1.7 \AA$ resolution (Fig. 1). The crystallographic structure was refined to an $R$-value of $20.7 \%$ ( $R_{\text {free }} 25.6 \%$ ) using all reflections between 26.8 and $1.7 \AA$ (for a detailed view of data collection and refinement statistics please refer to Table 1). A detailed view of the final electron density map is shown in Fig. 2A highlighting the overall quality of the experimental data. The asymmetric unit contains two molecules of $\mathrm{rBbCI}$ and 239 solvent molecules. The $\mathrm{rBbCI}$ structure presents a typical " $\beta$-trefoil" fold, as shown in Fig. 1. It is formed by six two-stranded hairpins, three of these form a barrel structure, and the other three are in a triangular array that caps the barrel. The

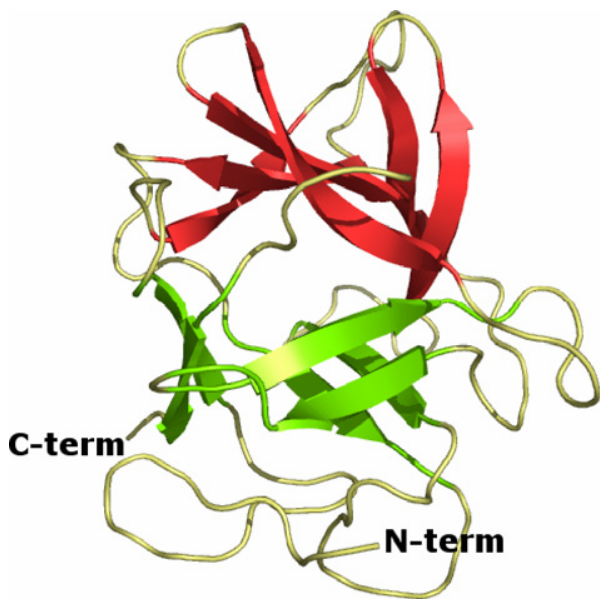

Fig. 1. The overall structure of $\mathrm{rBbCI}$. rBbCI has a $\beta$-trefoil fold, formed by six two-stranded hairpins. Three of these form a barrel structure (in green) and the other three are in a triangular array that caps the barrel (in red). (For interpretation of the references in color in this figure legend, the reader is referred to the web version of this article.)

Table 1

Data collection and refinement statistics

\begin{tabular}{|c|c|}
\hline Data collection & Monoclinic \\
\hline Group space & $\mathrm{P} 2_{1}$ \\
\hline Resolution $(\AA)$ & $26.82-1.7(2.06-1.7)^{\circ}$ \\
\hline Unit-cell parameters $\left(\AA{ }^{\circ}{ }^{\circ}\right)$ & $\begin{array}{l}a=61.96, b=41.32 \\
c=63.53, \beta=98.46\end{array}$ \\
\hline$R_{\mathrm{sym}}^{\mathrm{b}}(\%)$ & $8.2(43.4)^{\mathrm{a}}$ \\
\hline Number of observations & 505658 \\
\hline Unique reflections & 37751 \\
\hline Completeness (\%) & $95.2(91.9)^{\mathrm{a}}$ \\
\hline Rmerge (\%) & $8.2(43.4)$ \\
\hline$I / \sigma(\mathrm{I})$ & $7.0(1.7)$ \\
\hline \multicolumn{2}{|l|}{ Refinement } \\
\hline Final $R^{\mathrm{c}}, R_{\text {free }}(F>0)$ & $0.207,0.256$ \\
\hline Number of of residues per monomer & 163 \\
\hline Number of solvent molecules & 239 \\
\hline Average $B$ values ( $\AA 2$ ): Protein & 19.11 \\
\hline Average $B$ values (Å2): Water & 29.56 \\
\hline R.m.s.d. bonds $(\AA)$ & 0.022 \\
\hline R.m.s.d. angles $\left({ }^{\circ}\right)$ & 1.98 \\
\hline \multicolumn{2}{|l|}{ Ramachandran plot (\%) } \\
\hline Residues in most favorable regions & 92.2 \\
\hline Residues in additional allowed regions & 7.4 \\
\hline Residues in generously allowed regions & 0.4 \\
\hline Residues in disallowed regions & 0 \\
\hline
\end{tabular}

${ }^{a}$ Numbers in parentheses refer to the highest resolution shell.

${ }^{\mathrm{b}} R$ sym $=\sum|I-(I)| / \sum \mid .(I)$ is the average intensity of symmetry-related observations of a unique reflection.

${ }^{\mathrm{c}} R=\sum\left|F_{\mathrm{o}}-F_{\mathrm{c}}\right| / \sum F_{\mathrm{o}} . R_{\text {free }}$ is calculated as $R$ but for $10 \%$ of the reflections excluded from all refinement.

arrangement of the secondary structure gives the molecule a pseudo 3-fold axis. The amino acid Asp16, in both molecules in the asymmetric unit, is present in generously allowed region of the Ramachandran map [25], although it is perfectly well defined in the electron density map. This can be explained by the presence of a strong crystalline contact with the region of the loop formed with the amino 

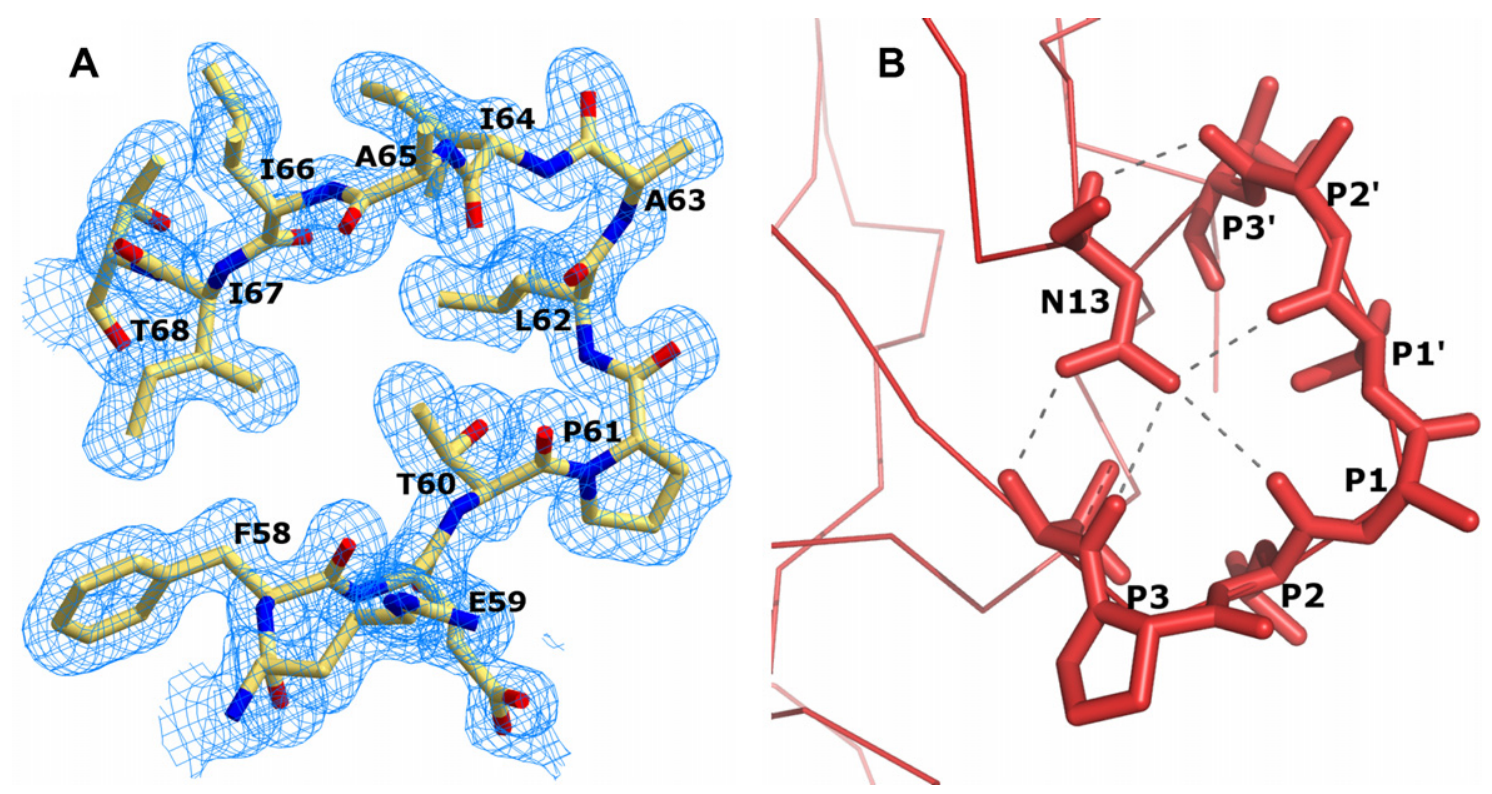

Fig. 2. (A) Electron density map, contoured at $1 \sigma$, of the $\mathrm{rBbCI}$ reactive site loop showing alanine 63 at position P1. (B) Residue Asn 13 forms a network of hydrogen bonds (dashed lines) scaffolding along with the reactive site loop (P3-P3́) of rBbCI.

acids Ser81, Ser82 and Asp83 of the symmetrically equivalent molecule.

\section{Structural comparison of rBbCI with other plant Kunitz-type inhibitors}

Despite their low sequence homology, crystallographic analyses of the Kunitz inhibitors from soybean [24], and Erythrina caffra [23], the interleukins-1 $\beta$ and $1 \alpha$ [26] and the acid and basic fibroblast growth factors [27] have shown that they share this unusual fold. BbCI shares a high structural homology with the plant Kunitz-type inhibitors BbKI, SbTI and ETI. The inhibitors rBbCI and rBbKI are $18 \mathrm{kDa}$ proteins with high structural identity and contain conserved residues characteristics of the plant Kunitztype inhibitor family, for example Asp5, Gly8, Pro10, Tyr17, Tyr18, Gly28 and Gly35/Asn36/Glu37 (SbTI numbering), but they do not contain any disulfide bridges. The residues Cys40, Cys80, Cys125 and Cys132 are substituted by Glu40, Ser80, Tyr125 and Gly132 in both rBbCI and rBbKI structures, as shown in Fig. 3. The superposition of the structurally equivalent $\mathrm{C} \alpha$ atoms of rBbCI with rBbKI and ETI gives an r.m.s. deviation of 0.88 and $1.9 \AA$ A, respectively. The central scaffold is common and larger deviations occur within the surface loops where overall sequence conservation is lower.

The major differences in $\mathrm{rBbCI}$ and $\mathrm{rBbKI}$ threedimensional structures is the loop which, in the case of $\mathrm{rBbCI}$, is formed by the amino acids Val78, Pro79, Ser80, Ser81, Ser82, Asp83, Ser84, Glu85 and in rBbKI by Gly78, Pro79, Ser80, Ser81, Ser82, Asp83, Ser84, Gly85 (Fig. 3). The change of Val78 and Glu85 in rBbCI to glycine in rBbKI permits different orientations in this loop-80. rBbKI loop is more flexible while in $\mathrm{rBbCI}$ the loop shows less mobility and is closer to the main core of the protein.

\section{Structural consequences of disulfide bond replacement}

In the classical Kunitz inhibitors such STI and ETI, the two disulfide bridges are localized on the surface of the protein, what makes them highly solvent-accessible, but the contribution of these structures on the maintenance of their functionality are on dependence of individual features of each protein. Thus, analysis of structural differences at this position may contribute to understand the significance of the disulfide bonds for the stability and inhibitory specificity of the proteins. Studies which had examined the role of disulfide bonds in the structure and unfolding of STI showed that the reduction of the disulfide bonds results in a fully inactive protein [28]. This result is in contrast to that shown in ETI, which remains fully active in both the disulfide intact and reduced states [29]. Thus, the disulfide bonds in STI play a crucial role in protein structure stabilization necessary to maintain enzymatic activity [28].

The absence of disulfide bridges in $\mathrm{rBbCI}$ and $\mathrm{rBbKI}$ might result in unfolded proteins and consequent loss of $\beta$-sheet structure. However, the replacement of Cys39, Cys83, Cys132 and Cys139 (ETI numbering, IDE3_ERYCA in Fig. 3) by Glu40, Ser80, Tyr125 and Gly 132 in $\mathrm{rBbCI}$ and $\mathrm{rBbKI}$, and which allowed the formation of hydrogen bonds, prevented a hydrophobic collapse of the protein, as it occurs with STI after the disulfide bonds were reduced. Tyr125 residue appears to be important for stabilization of $\mathrm{rBbCI}$ and $\mathrm{rBbKI}$ since the loop conformation is constrained by a water-mediated hydrogen bond between the Tyr125 and Gly132 


\begin{tabular}{|c|c|c|}
\hline IDE3 ERYCl & 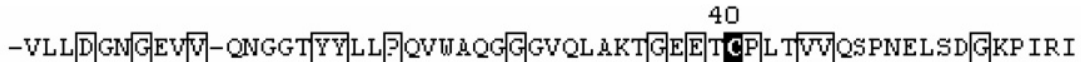 & 58 \\
\hline DE3_ER & GGT YYLL & 58 \\
\hline E3_ERYVR & QSPNELSNC & 58 \\
\hline IT1B_ERYVR & SRNDVSN & 58 \\
\hline ITRY_BAUVR & QASSEHSDG & 60 \\
\hline IFXA_BAUUN & SSE ISNG̈ & 59 \\
\hline $\mathrm{rBbKI}$ & PHHRP-GL & 58 \\
\hline rBbcI & PHHRP-GL & 58 \\
\hline & 63 & \\
\hline IDE3_ERYCl & ESTQF D YP F KF & 117 \\
\hline IDE3_ERYLR & DESTQF DYPF & 117 \\
\hline IDE3_ERYVR & DESTQF DYP $\mathrm{FF}$ & 117 \\
\hline IT1B_ERYVR & YESTEFNYS $\mid$ KF & 117 \\
\hline ITRY_BAUVR & S-DERFPLEF & 118 \\
\hline IFXA_BAUUN & $V$-DERFPLE & 117 \\
\hline $\mathrm{rBbK \overline {I }}$ & D--TKSLLGP $\mid \mathrm{FV}$ & 113 \\
\hline rBbcI & ETPL & 113 \\
\hline IDE3_ERYCl & VTEDYF: TVVLKKDESS- & 172 \\
\hline IDE3_ERYL & INRDQKGYRR LVTEDNF: TVVLKKDESS- & 172 \\
\hline IDE3_ERYVR & TTEDNF- TVVL KKDESS- & 172 \\
\hline IT1B_ERYVR & TNEENE:-VVNLKKVESS- & 176 \\
\hline ITRY_BAUVR & SDSCRDL GIS IDEEGNRR LVRDEN & 172 \\
\hline IFXi__BAUUN & -SSDSCRDLGIA IDEENNRR LVRDGKF _LVRF KE ANQDS & 171 \\
\hline $\mathrm{BbK \overline {I }}$ & QTGLD I GLLHRNDKYY- & 164 \\
\hline rBbcI & PVVFIRK & 164 \\
\hline
\end{tabular}

Fig. 3. The amino acid sequence alignment of $\mathrm{rBbCI}$ and $\mathrm{rBbKI}$ inhibitors with representative members of the plant-type Kunitz inhibitor family. The alignment was performed using ClustalW [43] (http://www.ebi.ac.uk/clustalw). Conserved amino acids are boxed in white the cysteine residues are within a black box and the amino acid in P1 is highlighted by a circle.

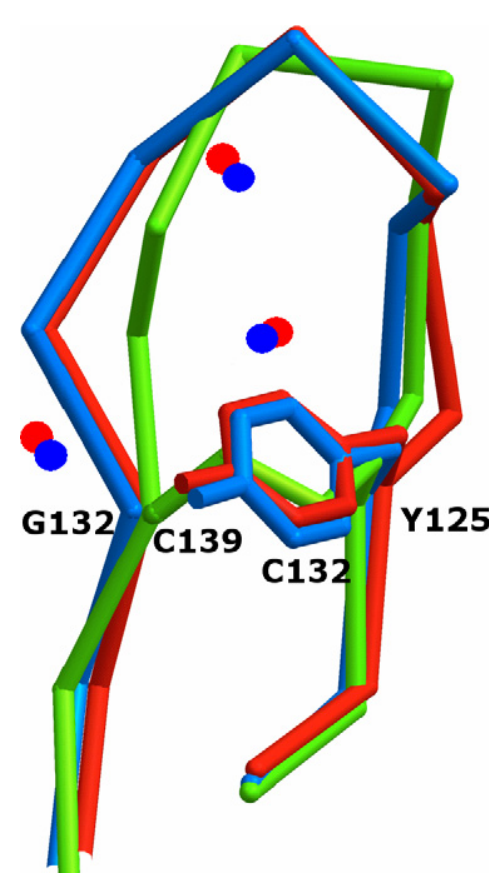

Fig. 4. A water-mediated hydrogen bond in Bauhinia inhibitors replace the disulfide bridges formed between Cys132 and Cys139 in ETI (green) $\mathrm{rBbCI}$ is shown in red and $\mathrm{rBbKI}$ in blue. The figures were prepared with the program Pymol [44]. (For interpretation of the references in color in this figure legend, the reader is referred to the web version of this article.)
(Fig. 4). This interaction replaces the ETI disulfide bridges involving residues Cys132 and Cys139 (ETI numbering). In the case of $\mathrm{rBbCI}$ and $\mathrm{rBbKI}$, our studies clearly demonstrate that polar interaction of the protein is involved in the maintenance of the structural folding and therefore in the inhibitory function.

The other disulfide bridge, Cys39-Cys83 (ETI numbering), also common in classical plant-type Kunitz inhibitors, apparently reduces the flexibility of the loops by cross-linking the loops (loop-40 and loop-80) containing these residues. In both rBbCI and rBbKI, as a result of the missing disulfide bond, loop- 80 moves away from loop40 , approaching the reactive site loop- 60 . Loop- 80 is, the most divergent loop when compared to the structure of ETI.

Within BbKI loop-80, hydrogen bonds between Ser80 and Gly14 help to stabilize both loops, maintaining Asn13 in position to form a hydrogen bond network in the reactive site loop. In $\mathrm{rBbCI}$, Ser80 forms a hydrogen bond with Ser82, within the same loop. The presence of the Gly14 in rBbKI and not in rBbCI confers higher flexibility to this loop, flexibility that is compensated in rBbKI by formation of a strong H-bond with Ser80.

In $\mathrm{rBbCI}$, both loops 80 and 14 contain less glycine residues, therefore they are more rigid, loop- 80 is internally stabilized by contacts with Ser82 and loop-14 is stabilized by a H-bond with the $\mathrm{CO}$ of Phe58 [14]. 


\section{Comparison of the $r B b C I, r B b K I$ and ETI reactive site}

The geometry of the carbonyl group at the $\mathrm{P} 1$ position is relevant to generate an interaction between inhibitor and protease during catalysis $[30,31]$. The residues adjacent to P1 contribute to the inhibitory selectivity of the enzyme due to the characteristics of each amino acid establishing others interactions, such as hydrogen and saline bridges or steric influences that are fundamental to the inhibitory interaction. Alanine 63, identified as the P1 residue in the putative reactive site of $\mathrm{rBbCI}$ was confirmed by $\mathrm{HNE}$ and cruzipain/cruzain specific cleavage [11,14], as shown in Fig. 2A and B.

The maintenance of the canonical conformation of the reactive site loop is also considerable for the proper inhibitory function and protein scaffold plays a role at this position. Owing to its crucial position, the role of a conserved Asn (Asn13 in rBbCI and rBbKI and Asn12 in ETI) was emphasized in the Kunitz family of inhibitors [23,24,31]. In $\mathrm{rBbCI}$ crystallographic structure also keeps the stability, in addition with other interactions, of the loop formed between Thr60, Pro61, Leu62, Ala63, Ile64 and Ala65 of the reactive site, forming a hydrogen bond network, as shows Fig. 4.

Studies have pointed out the positive contribution of the hydrophobic characteristic of alanine at the $\mathrm{P} 1$ position for the interaction to the subsite $\mathrm{S} 1$ of elastase $[32,33]$ and of cruzain [34]. Thus, the residue at $\mathrm{P} 1$ position of $\mathrm{rBbCI}$ reactive-site (Ala63) was mutated to examine its role in the inhibitory specificity. It was shown that this residue is crucial for elastase and cruzain inhibitory activity since rBbCI-A63R did not affect the enzyme substrate binding. However, the mutant inhibitor acquired the property for trypsin inhibition measured by the association constant, which is in the same order of magnitude than that of rBbKI $\left(\mathrm{Ki}_{\text {app }} 28 \mathrm{nM}\right)$ [14], thereby supporting their reactive-site integrity and its role in the strict selectivity of $\mathrm{BbCI}$, which is able to inhibit the serine proteases elastase and cathepsin $\mathrm{G}$ but not trypsin and trypsin like proteases.

\section{Materials and methods}

Crystallization. The $\mathrm{rBbCI}$ was obtained as described previously [14]. Crystals were grown by vapor diffusion at $18{ }^{\circ} \mathrm{C}$ temperature using the hanging drop method, in two different crystallization buffers: $0.1 \mathrm{M}$ sodium acetate, $\mathrm{pH} 8.6$ containing $0.2 \mathrm{M}$ ammonium acetate and polyethylene glycol 4000 (30\%), (rBbCI monoclinic) and $0.1 \mathrm{M}$ Hepes, $\mathrm{pH} 7.5$ containing $1.5 \mathrm{M}$ lithium sulphate ( $\mathrm{rBbCI}$ triclinic). Diffraction data were collected using a RIGAKU ultra X 18 rotating anode with a MAR345 dtb image plate. The resulting crystal (rBbCI monoclinic) belonged to space group $p 2_{1}$ with cell dimensions of $a=61.96 \AA, b=41.32 \AA, c=63.53 \AA$, $\beta=98.46^{\circ}$ and with two inhibitor molecules, related by a non-crystallographic 2-fold symmetry axis, in unit asymmetric, which was the main object of this work (data shown in Table 1).

$\mathrm{rBbCI}$ triclinic crystals (space group $\mathrm{p} 321$ ) diffracted to lower resolution and data were not further used.

Data collection, processing, phasing, and refinement. $\mathrm{rBbCI}$ monoclinic data were collected at IFSC Crystallography Laboratory to $1.70 \AA$ А resolution and processed using the programs MOSFLM [35] and SCALA $[36,37]$ from the CCP4 package [38].
The structure was solved by molecular replacement using the program MOLREP [39] and the crystal structure of rBbKI (recombinant Bauhinia bauhinioides Kallikrein Inhibitor) as a search model (PDB ID code 2GO2), both structures were solved at the same time, but rBbKI structure was determined by SIRAS [15]. Alternate rounds of model building with the program $\mathrm{O}$ [40] and further refinement with the program Refmac 5.0 [41] resulted in the final model with an $R_{\text {free }}$ an $R_{\text {factor }}$ of 25.6 and $20.7 \%$ for all data between 26.8 and $1.70 \AA$ resolutions, respectively. The model was analyzed with Procheck [42] and showed $92.2 \%$ of the residues in core region of the Ramachandran plot [25], while the remaining $7.4 \%$ were present in the additionally allowed region. A single residue (Asp16) lies in the generously allowed region in both molecules A and B, but it is perfectly well defined in the electron density map. Coordinates have been deposited in the RCSB Protein Data Bank: PDB ID code 2GZB.

BbCI mutagenesis. The reactive site P1 residue encoding Ala63 was mutated to encode Arg63 through site-directed mutagenesis using a QuikChange Site-Directed Mutagenesis Kit (Stratagene, Germany) (BbCI-A63R) where the pET28aBbCI DNA double-stranded and $100 \mathrm{pmol}$ of each primer containing the mutation desired (BbCIA63R_FOWARD: 5'-CGG TTA GAT TTG AAA CCC CAC TTC GCA TTG CTA TAA TCA CAG AGT C-3' BbCI-A63R_REVERSE: 5'-GAC TCT GTG ATT ATA GCA ATG CGA AGT GGG GTT TCA AAT CTA ACC G-3') were used. The mutant protein rBbCI-A63R was expressed in Escherichia coli BL21 (DE3) and its specificity was assayed on cruzain, PPE and trypsin activity as described previously for $\mathrm{rBbCI}$ $[8,11,12,14]$.

\section{Acknowledgments}

The authors thank Dr. Richard C. Garratt, Dr. Ana Paula U. Araújo and Dr. Marcos V. Navarro from Instituto de Física de São Carlos, USP for diffraction data collection and for helpful discussions. CAPES/DAAD/Grices, CNPq, FAPESP and SPDM/FADA provided financial assistance.

\section{References}

[1] M. Richardson, Seed storage proteins: the enzyme inhibitors. Methods in plant, Biochemistry 5 (1991) 259-305.

[2] Y. BirkIn Plant Protease Inhibitors: Significance in Nutrition, Plant Protection, Cancer Prevention and Genetic Engineering, SpringerVerlag, Berlin Heidelberg, 2003, pp. 1-126.

[3] A. Soares-Costa, L.M. Beltramini, O.H. Thiemann, F. HenriqueSilva, A sugarcane cystatin: recombinant expression purification \& antifungal activity, Biochem. Biophys. Res. Commun. 296 (2002) 1194-1199.

[4] C.A. Ryan, The systemin signaling pathway: differential activation of plant defensive genes, Biochim. Biophys. Acta 1477 (2000) $112-121$.

[5] D.C. Damasceno, G.T. Volpato, I.M. Calderon, R. Aguilar, M.V. Rudge, Effect of Bauhinia forficata extract in diabetic pregnant rats: maternal repercussions, Phytomedicine 11 (2004) 196-201.

[6] O.L. Zucchi, S. Moreira, E. F de Jesus, H.S. Neto, M.J. Salvador, Characterization of hypoglycemiant plants by total reflection X-ray fluorescence spectrometry, Biol. Trace Elem. Res. 103 (2005) 277-290.

[7] A. Pott, V.J. Pott, Plantas do Pantanal. Embrapa, Corumbá, 1994, pp. 320.

[8] C. Oliveira, L.A. Santana, A.K. Carmona, M.H. Cezari, M.U. Sampaio, C.A.M. Sampaio, M.L.V. Oliva, Structure of Cruzipain/ Cruzain Inhibitors isolated from Bauhinia bauhinioides seeds, Biol. Chem. 382 (2001) 847-852.

[9] A.E. Eakin, A.A. Mills, G. Harth, J.H. McKerrow, C.S. Craik, The sequence, organization, and expression of the major cysteine protease (cruzipain) from Trypanosoma cruzi, J. Biol. Chem. 267 (1992) 74117420. 
[10] C.J. Schofield, J. Jannin, R. Salvatella, The future of Chagas' disease control, Trends Parasitol. 22 (2006) 583-588.

[11] C. Neuhof, M.L. Oliva, D. Maybauer, M. Maybauer, C. Oliveira, M.U. Sampaio, C.A. Sampaio, H. Neuhof, Effect of plant Kunitz inhibitors from Bauhinia bauhinioides and Bauhinia rufa on pulmonary edema caused by activated neutrophils, Biol. Chem. 384 (2003) 939-944.

[12] M.L. Oliva, C.R. Mendes, M.A. Juliano, J.R. Chagas, J.C. Rosa, L.J. Greene, M.U. Sampaio, C.A.M. Sampaio, Characterization of a tissue kallikrein inhibitor isolated from Bauhinia bauhinioides seeds: inhibition of the hydrolysis of kininogen related substrates, Immunopharmacology 45 (1999) 163-169.

[13] M.V. Navarro, D.F. Vieira, R.A. Nagem, A.P. de Araújo, M.L. Oliva, R.C. Garratt, Crystallization and preliminary X-ray analysis of a novel Kunitz-type kallikrein inhibitor from Bauhinia bauhinioides, Acta Crystallogr. Sect. F Struct. Biol. Crystallogr. Commun. 61 (2005) 910-913.

[14] A.P.U. Araújo, D. Hansen, D.F. Vieira, C. Oliveira, L.A. Santana, L.M. Beltramini, C.A. M Sampaio, M.U. Sampaio, M.L.V. Oliva, Kunitz type Bauhinia bauhinioides inhibitors devoid of disulfide bridges: isolation of the cDNAs, heterologous expression and structural studies, Biol. Chem. 386 (2005) 561-568.

[15] M.C. Do Socorro, M.L.V. Oliva, H. Fritz, M. Jochum, R. Mentele, M.U. Sampaio, L.C.B.B. Coelho, I.F.C. Batista, C.A.M. Sampaio, Characterization of a Kunitz Trypsin Inhibitor with one disulfide bridge purified from Swartzia pickellii, Biochem. Biophys. Res. Commun. 291 (2002) 635-639.

[16] J.T. Sumikawa, A.M. Nakahata, H. Fritz, R. Mentele, M.U. Sampaio, M.L.V. Oliva, A Kunitz-type glycosylated elastase inhibitor with one disulfide bridge, Planta Med. 72 (2006) 393-397.

[17] A.M. Nakahata, N.R. Bueno, H.A. Rocha, C.R. Franco, R. Chammas, C.R. Nakaie, M.G. Jasiulionis, H.B. Nader, L.A. Santana, M.U. Sampaio, M.L. Oliva, Structural and inhibitory properties of a plant proteinase inhibitor containing the RGD motif, Int. J. Biol. Macromol. 40 (2006) 22-29.

[18] V. Turk, Review: targeting proteases: successes, failures and future prospects, Nat. Rev. 5 (2006) 785-799.

[19] C.A. Orengo, D.T. Jones, J.M. Thornton, Protein superfamilies and domain superfolds, Nature 372 (1994) 631-634.

[20] D. Mukhopadhyay, The molecular evolutionary history of a winged bean $\alpha$-Chymotrypsin Inhibitor and modelling of its mutations through structural analises, J. Mol. Evol. 50 (2000) 214-223.

[21] A.G. Murzin, A.M. Lesk, C. Chothia, $\beta$-trefoil fold. Patterns of structure \& sequence in the Kunitz Inhibitors, interleukins-1(and 1-(and fibroblast growth factors, J. Mol. Biol. 223 (1992) 531-543.

[22] R. Xu, Y. Xiau, A common sequence-associated physicochemical feature for proteins of beta-trefoil family, Comput. Biol. Chem. 29 (2005) 79-82.

[23] S. Onesti, P. Brick, D.M. Blow, Crystal structure of a Kunitz-type Tripsin Inhibitor from Erythrina caffra seeds, J. Mol. Biol. 217 (1991) 153-176.

[24] H.K. Song, S.W. Suh, Kunitz type Soybean Trypsin Inhibitor revisited: refined structure of its complex with porcine trypsin reveals an insight into the interaction between a homologous inhibitor from Erythrina caffra and tissue-type plasminogen activator, J. Mol. Biol. 275 (1998) 347-363.

[25] G.N. Ramachandran, V. Sasisekharan, Conformation of polypeptides and proteins, Adv. Protein Chem. 23 (1968) 383-438.
[26] B.J. Graves, M.H. Hatada, W.A. Hendrickson, J.K. Miller, V.S Madison, Y. Satow, Structure of interleukin 1 at 2.7 angstrom resolution, Biochemistry 29 (1990) 2679-2684.

[27] H. Ago, Y. Kitagawa, A. Fujishima, Y. Matsura, Y. Katsube, Crystal structure of basic fibroblast growth factor at $1.6 \AA$ resolution, J. Biochem. (Tokyo) 110 (1991) 360-363.

[28] J. Tetenbaum, L.M. Miller, A new spectroscopy approach to examining the role of disulfide bonds in the structure and unfolding of soybean trypsin inhibitor, Biochemistry 40 (2001) 12215-12219.

[29] K. Lehle, A. Wrba, R. Jaenicke, Erythrina caffra trypsin inhibitor retains its native structure and function after reducing its disulfide bonds, J. Mol. Biol. 239 (1994) 276-284.

[30] R. Sattar, S.A. Ali, M. Kamal, A.A. Khan, A. Abbasi, Molecular mechanism of enzyme inhibition: prediction of the three-dimensional structure of the dimeric trypsin inhibitor from Leucaena leucocephala by homology modelling, Biochem. Biophys. Res. Commun. 314 (2004) 755-765.

[31] S. Khamrui, J. Dasgupta, J.K. Dattagupta, U. Sem, Single mutation at $\mathrm{P} 1$ of a chymotrypsin inhibitor changes it to a trypsin inhibitor: $\mathrm{X}$-Ray structural $(2.15 \AA)$ and biochemical basis, Biochimica et Biophysica Acta 1752 (2005) 65-72.

[32] M.A. Navia, B.M. McKeever, J.P. Springer, T.Y. Lin, H.R. Willians, E.M. Fluder, C.P. Dorn, K. Hoogsteen, Structure of human neutrophil elastase in complex with a peptide chloromethyl ketone inhibitor at $1.84 \AA$ resolution, Biochemistry 86 (1989) 7-11.

[33] M. Tsunemi, Y. Matsuura, S. Sakakibara, Y. Katsube, Crystal structure of an Elastase-specific Inhibitor Elafin complexed with porcine pancreatic Elastase determined at $1.9 \AA$ A resolution, Biochemistry 35 (1996) 11570-11576.

[34] L.C. Alves, R.L. Melo, S.J. Sanderson, J.C. Mottram, G.H. Coombs, G. Caliendo, V. Santagada, L. Juliano, M.A. Juliano, S1 subsite specificity of a recombinant cysteine protease, CPB, of Leishmania mexicana compared with cruzain, human cathepsin $\mathrm{L}$ and papain using substrates containing non-natural basic amino acids, Eur. J. Biochem. 268 (2001) 1206-1212.

[35] A.G.W. Leslie, Joint CCP4 and ESF-EACMBNewsletters on Protein Crystallography, Vol. 26, Daresbury Laboratory, Warrington, UK, 1992.

[36] W. Kabsch, Evaluation of single-crystal X-ray diffraction data from a position-sensitive detector, J. Appl. Crystallogr. 21 (1988) 916-924.

[37] R.H. Blessing, An empirical correction for absorption anisotropy, Acta. Crystallogr. A51 (1995) 33-38.

[38] Collaborative Computational Project, Number 4, The CCP4 suite: programs for protein crystallography, Acta. Crystallogr. D50 (1994) $760-763$.

[39] A. Vagin, A. Teplyakov, MOLREP: an automated program for molecular replacement, J. Appl. Crystallogr. 30 (1997) 1022-1025.

[40] T.A. Jones, J.Y. Zou, S.W. Cowan, M. Kjeldgaard, Improved methods for building protein models in electron density maps and the location of errors in these models, Acta Crystallogr. A47 (1991) 110 119 .

[41] G.N. Murshudov, A. A Vagin, E.J. Dodson, Acta Crystallogr. D53 (1997) 240-255.

[42] R.A. Laskowski, M.W. MacArthur, D.S. Moss, J.M. Thornton, PROCHECK: a program to check the stereochemical quality of protein structures, J. Appl. Crystallogr. 26 (1993) 283-291.

[43] W.R. Pearson, Rapid and sensitive sequence comparison with FASTP and FASTA, Methods Enzymol. 183 (1990) 63-98. 